\title{
Diagnostic Accuracy of an MRI Protocol of the Knee Accelerated Through Parallel Imaging in Correlation to Arthroscopy
}

\author{
Diagnostische Genauigkeit eines mittels paralleler \\ Bildgebung beschleunigten Knie-MRT-Protokolls in \\ Korrelation zur Arthroskopie
}

\section{Authors}

Johannes Walter Schnaiter ${ }^{1}$, Frank Roemer ${ }^{2}$, Axel McKenna-Kuettner ${ }^{1}$, Hans-Joachim Patzak ${ }^{3}$, Matthias Stefan May², Rolf Janka², Michael Uder ${ }^{2}$, Wolfgang Wuest ${ }^{2}$

\author{
Affiliations \\ 1 Radiology, Community Practice of Radiology and Nuclear \\ Medicine, Bad Nauheim, Germany \\ 2 Radiological Institute, Friedrich-Alexander-University \\ Erlangen-Nürnberg, Erlangen, Germany \\ 3 Surgery, Sports Clinic, 61231 Bad Nauheim, Germany
}

Key words

cartilage, surgery, skeletal-axial, extremities, bones, MR imaging

received 07.05.2017

accepted 12.08.2017

Bibliography

DOI https://doi.org/10.1055/s-0043-119038

Published online: 26.9.2017

Fortschr Röntgenstr 2018; 190: 265-272

(c) Georg Thieme Verlag KG, Stuttgart · New York

ISSN 1438-9029

Correspondence

Johannes Walter Schnaiter

Standort Bad Nauheim, Gemeinschaftspraxis für Radiologie und Nuklearmedizin, In der Au 30-32, 61231 Bad Nauheim, Germany

Tel.: ++ 49/69/7580860

Fax: $++49 / 69 / 75808630$

johannes.schnaiter@hotmail.com

\section{ABSTRACT}

Purpose Parallel imaging allows for a considerable shortening of examination times. Limited data is available about the diagnostic accuracy of an accelerated knee MRI protocol based on parallel imaging evaluating all knee joint compartments in a large patient population compared to arthroscopy. Materials and Methods 162 consecutive patients with a knee MRI (1.5T, Siemens Aera) and arthroscopy were included. The total MRI scan time was less than 9 minutes. Meniscus and cartilage injuries, cruciate ligament lesions, loose joint bodies and medial patellar plicae were evaluated. Sensitivity (SE), specificity (SP), positive predictive value (PPV), and negative predictive value (NPV), as well as diagnostic accuracy were determined.

Results For the medial meniscus, the values were: SE $97 \%$, SP $88 \%$, PPV $94 \%$, and NPV $94 \%$. For the lateral meniscus the values were: SE $77 \%$, SP $99 \%$, PPV $98 \%$, and NPV $89 \%$. For cartilage injuries the values were: SE $72 \%$, SP $80 \%$, PPV $86 \%$, and NPV $61 \%$. For the anterior cruciate ligament the values were: SE $90 \%$, SP $94 \%$, PPV $77 \%$, and NPV $98 \%$, while all values were $100 \%$ for the posterior cruciate ligament. For loose bodies the values were: SE $48 \%$, SP $96 \%$, PPV $62 \%$, and NPV $93 \%$, and for the medial patellar plicae the values were: SE $57 \%$, SP $88 \%$, PPV $18 \%$, and NPV $98 \%$.

Conclusion A knee MRI examination with parallel imaging and a scan time of less than 9 minutes delivers reliable results with high diagnostic accuracy.

\section{Key Points}

- An accelerated knee MRI protocol with parallel imaging allows for high diagnostic accuracy.

- Especially meniscal and cruciate ligament injuries are well depicted.

- Cartilage injuries seem to be overestimated.

\section{Citation Format}

- Schnaiter JW, Roemer F, McKenna-Kuettner A et al. Diagnostic Accuracy of an MRI Protocol of the Knee Accelerated Through Parallel Imaging in Correlation to Arthroscopy. Fortschr Röntgenstr 2018; 190: 265-272

\section{ZUSAMMENFASSUNG}

Ziel Parallele Bildgebung ermöglicht eine deutliche Reduktion der Untersuchungszeit, allerdings gibt es kaum Daten über die diagnostische Genauigkeit eines auf der Basis paralleler Bildgebung beschleunigten Protokolls an einem größeren Patientengut, in dem alle Kniegelenkskompartimente im direkten Vergleich zur Arthroskopie als Goldstandard verglichen wurden. 
Material und Methoden Insgesamt wurden 162 Patienten mit einer Knie-MRT (1.5 T, Siemens Aera) und anschließender Arthroskopie in die Studie eingeschlossen. Die MRT-Gesamtscanzeit betrug $<9$ min. Beurteilt wurden Meniskus- und Knorpelschäden, Kreuzbandläsionen, freie Gelenkkörper (FGK) sowie Plicae mediopatellares (PM). Sensitivität (SE), Spezifität (SP), positiver Vorhersagewert (PPV) und negativer Vorhersagewert (NPV), und die Genauigkeit einer korrekten Beurteilung aller Pathologien wurden bestimmt.

Ergebnisse Für Innenmeniskusschäden ergab sich eine SE von $97 \%$, eine SP von $88 \%$, ein PPV von $94 \%$ und ein NPV von $94 \%$, für den Außenmeniskus eine SE von $77 \%$, eine SP von $99 \%$, ein PPV von $98 \%$ und ein NPV von $89 \%$. Bezüglich der Knorpelschäden ergaben sich eine SE von $72 \%$, eine SP von $80 \%$, ein PPV von $86 \%$ und ein NPV von $61 \%$. Für das vordere Kreuzband betrug die SE $90 \%$, die SP $94 \%$, der PPV $77 \%$ und der NPV $98 \%$, für das hintere Kreuzband ergaben sich jeweils $100 \%$. Bei FGK betrug die SE $48 \%$, die SP $96 \%$, der PPV $62 \%$ und der NPV $93 \%$ und für die PM ergab sich eine SE von $57 \%$, eine SP von $88 \%$, ein PPV von $18 \%$ und ein NPV von $98 \%$.

Schlussfolgerung Mithilfe paralleler Bildgebung liefert eine Knie-MRT Untersuchung verlässliche Ergebnisse von hoher diagnostischer Wertigkeit bei einer Gesamtscanzeit von $<9$ min.

\section{Introduction}

Knee MRI provides high accuracy for the detection of traumatic and degenerative internal knee injuries [1-8]. MRI is established in the clinical routine as a noninvasive method for evaluating acute knee trauma and for visualizing possible internal knee injuries. The clinical role of MRI in the evaluation of gonarthrosis is less clearly defined but it is frequently used to rule out loose joint bodies and unstable meniscus tears, for example in recurrent locking of the knee joint.

Technical advances in recent years have made it possible to quickly acquire high-resolution images. MRI systems with higher magnetic field strengths, high-performance gradients, and the use of multichannel coils and pulse sequences with parallel imaging and thus a shorter acquisition time are largely responsible for this [9-11]. During parallel imaging, raw data are acquired simultaneously via two or more receiver coils. By arranging the coil elements in the phase-encoding direction, the number of phase-encoding steps can be reduced thus accelerating the scan time. Faster MRI protocols are interesting from an economic standpoint and also minimize motion artifacts as a result of the shorter scan times. The disadvantages of the parallel acquisition technique are a reduced signal-to-noise ratio and possible artifacts in the case of an imperfect image reconstruction algorithm [12]. Previous studies analyzing the diagnostic value of an accelerated MRI protocol with parallel imaging for evaluating changes to the knee joint frequently used the conventional protocol as a reference standard [12-14].

The goal of this study is to determine the diagnostic value of a time-optimized MRI protocol using parallel imaging with a total scan time of less than 9 minutes compared to arthroscopy as the reference standard in patients with traumatic or degenerative knee problems.

\section{Materials and Methods}

\section{Patient population}

In total, 706 consecutive patients were examined with the timeoptimized MRI protocol. 162 of these patients, including 97 men and 65 women with an age range of $17-77$ years, subsequently underwent arthroscopy and were included in the study. Arthroscopy was performed between 35 and 39 days after the MRI examination. The indication for arthroscopy was derived from the MRI finding. In the case of a discrepancy between the MRI finding and the patient's symptoms, the indication for arthroscopy was determined at the operator's discretion.

The results of the arthroscopy procedures were retrospectively compared to the MRI findings. Compliance with ethical guidelines was ensured in accordance with the Declaration of Helsinki. Due to the retrospective design and complete anonymization of the data, a formal application for approval from the ethics committee was not submitted and the written approval of the institutional review board (IRB) was not obtained.

\section{MRI protocol}

The examinations were performed on a 1.5 T MRI scanner (Magnetom Aera, Siemens Healthineers, Erlangen) using a 15-channel phased-array knee coil (TxRx15 ChKnee from Siemens). A 4-sequence protocol with parallel imaging (GRAPPA, acceleration factor 2) and the parameters listed in detail in $>$ Tab. 1 were used. The scan time was $<9$ min.

\section{Arthroscopy}

All arthroscopy procedures were performed by an experienced trauma surgeon (approx. 700 arthroscopy procedures/year, in total approx. 16000 arthroscopy procedures). The surgeon was not blinded to the results of the previously performed MRI examination. The joint was accessed ventrally (anterolateral and anteromedial access with a $30^{\circ}$ angled lens). Per video transmission, the medial, lateral, intercondylar, and retropatellar compartments were examined and evaluated with respect to stability by means of a hook probe, and the condition of the menisci, cartilage and cruciate ligaments and the presence of medial patellar plicae and loose joint bodies in the above-mentioned compartments in relation to the radiological examination were documented preoperatively and postoperatively. After the diagnostic portion, the surgeon initiated therapeutic measures as necessary. 
- Table 1 Imaging parameters for MRI sequences.

\begin{tabular}{|l|l|l|l|l|}
\hline imaging parameter & Sagittal PD TSE FS & Coronal PD TSE FS & Axial PD TSE FS & Sagittal PD TSE \\
\hline TR (msec) & 3080 & 3000 & 3000 & 1300 \\
\hline TE (msec) & 41 & 38 & 37 & 44 \\
\hline Matrix size & $323 \times 384$ & $358 \times 448$ & $314 \times 448$ & $285 \times 320$ \\
\hline Field of view (mm) & 180 & 180 & 160 & 160 \\
\hline Section thickness (mm) & 3.5 & 3.5 & 3.5 & 2 \\
\hline Voxel size (mm $)$ & $0.5 \times 0.5 \times 3.5$ & $0.2 \times 0.2 \times 3.5$ & $0.4 \times 0.4 \times 3.5$ & 130 \\
\hline Bandwidth (Hz/Px) & 110 & 140 & 140 & 45 \\
\hline Echo train length & 46 & 47 & 20 & 1 \\
\hline Averages & 1 & 1 & 2 & $1: 01$ \\
\hline Imaging time (min) & $2: 26$ & $2: 29$ & $2: 16$ & 2 \\
\hline iPAT ${ }^{1}$ grappa & 2 & 2 & 2 & \\
\hline 1 Integrated parallel acquisition techniques. & & & \\
\hline 2 Generalized autocalibrating partially parallel acquisitions. & & & \\
\hline
\end{tabular}

\section{Evaluating the MRI scans}

The knee MRI images acquired in the clinical routine were retrospectively evaluated by a board-certified radiologist with more than 10 years of professional experience. This was a secondary evaluation based on standardized criteria following the initial clinical MRI finding. The menisci, cruciate ligaments, and articular cartilage in all knee joint compartments and the presence of loose joint bodies and medial patellar plicae were evaluated. The evaluator was blinded to the arthroscopic finding.

The menisci were evaluated on the basis of the diagnostic criteria according to Nguyen [15] and De Smet [16]. A tear was diagnosed according to the following criteria: Distortion of the meniscus or a signal increase reaching the joint surface on at least two images with a slice thickness of $3 \mathrm{~mm}$. The scans did not have to be consecutive. Two coronal scans, two sagittal scans, or also one coronal scan and one sagittal scan were permissible. A single image according to the indicated criteria or an intrameniscal signal increase not reaching the surface was evaluated as "no tear". Displaced meniscal fragments were considered a meniscus injury and were thus classified as a "tear" even though a linear signal increase reaching the surface on more than one image is not always seen in such patients [16].

The stages of chondropathy were defined based on the classification system of Noyes [17], with grade 0 corresponding to normal physiological cartilage and grade 1 to a signal alteration of morphologically intact cartilage. Grade $2 \mathrm{~A}$ indicated a superficial chondral defect $\leq 50 \%$ of the total chondral thickness, grade $2 \mathrm{~B}$ a deep chondral defect $>50 \%$ of the total chondral thickness and grade 3 chondral lesion extending to the subchondral bone ( $\triangleright$ Tab.2). The surface expansion was not taken into consideration in the classification system. Chondropathy was graded in 6 compartments: medial and lateral tibia, medial and lateral femur, retropatellar and trochlear groove. If it was not possible to defini- tively decide between two grades, the higher grade was always selected (e. g. grade $1-2 A=2 A$ ).

Circumscribed discontinuity, the complete absence of a cruciate ligament, abnormal signal intensity, a wavy contour or poor delineation of the ligament fibers was evaluated as a cruciate ligament tear. A partial cruciate ligament tear diagnosed on MRI was classified as a "cruciate ligament tear" in the study since stability cannot be determined based on MRI findings ( $>$ Tab. 3 ).

Loose joint bodies were considered to be present if at least one loose joint body was detected on MRI.

A "radiologically symptomatic" plica was determined on MRI in the case of the simultaneous presence of a plica and cartilage damage in a typical location on the medial patellar facet.

The MRI findings regarding the menisci, cruciate ligaments, loose joint bodies, and medial patellar plicae were evaluated again with knowledge of the arthroscopy results in order to differentiate method-related errors (e.g. structural lesion not shown on MRI) from evaluation-based errors (e.g. finding visible on MRI but not evaluated) in the indicated regions. However, the subsequently acquired data did not affect the previously performed statistical calculations.

\section{Statistical analysis}

The sensitivity (SE), specificity (SP), positive predictive value (PPV) and negative predictive value (NPV) were determined with respect to the detection of damage to the menisci, cruciate ligaments, and cartilage in all compartments and the presence of loose joint bodies and medial patellar plicae. The accuracy was expressed as the percentage of diagnoses correctly made on MRI compared to arthroscopy.

A difference of up to one grade compared to the arthroscopic finding was tolerated in the cartilage evaluation as a non-significant deviation. A Wilcoxon signed rank test was additionally performed to determine the correct grade of chondral damage. All 
- Table 2 Chondropathic stages based on Noyes [17] for radiologists and surgeons.

\begin{tabular}{|l|l|l|}
\hline & MRI Findings & Arthroscopy Findings \\
\hline Grade 0 & Without pathological findings & Without pathological findings \\
\hline Grade 1 & Superficial signal increase & Localized softening \\
\hline Grade 2A & Superficial chondral damage $\leq 50 \%$ of total chondral thickness & Massive fraying/maceration \\
\hline Grade 2B & Deep chondral damage $>50 \%$ of total chondral thickness & Grade $2 \mathrm{~A}+$ chondral loss/chondral fissures/instabilities \\
\hline Grade 3 & Chondral erosion of the subchondral bone & Chondral erosion of the subchondral bone \\
\hline
\end{tabular}

- Table 3 Findings of the cruciate ligament in radiologic (based on Robertson et al. [18]) and arthroscopic evaluation.

\begin{tabular}{|l|l|l|l|}
\hline & MRI Findings & Arthroscopy Findings & Study \\
\hline Grade I & No tear & No tear & "No tear of the cruciate ligament" \\
\hline Grade II & Partial tear & Stable partial damage & "Tear of the cruciate ligament" \\
\hline Grade III & Tear & Unstable in examination with hook probe & \\
\hline
\end{tabular}

statistical analyses were evaluated with a statistics program (SPSS Statistics, Version 20, SPSS Inc./ IBM, Chicago, IL).

\section{Results}

A detailed overview of the SE, SP, PPV, NPV and diagnostic accuracy of the shortened protocol regarding meniscus and cruciate ligament injuries, loose joint bodies and medial patellar plicae is provided in $>$ Tab. 4.

\section{Menisci}

$93 \%$ accuracy (300/324 correct diagnoses) was achieved with respect to the detection of meniscus injuries of the medial and lateral meniscus.

104 medial meniscus tears were detected by arthroscopy (prevalence $64 \%$ ). 101 of these were correctly detected on MRI, but 3 tears detected by arthroscopy could not be detected on MRI. Arthroscopy identified a lateral meniscus tear in 57 patients (prevalence $35 \%$ ). 44 of these tears were identified correctly on MRI ( $\triangleright$ Fig. 1), while 13 tears could not be diagnosed. When the MRI images were viewed again, all initially undetected medial meniscus tears still could not be identified despite knowledge of the arthroscopic findings. 3/13 undetected lateral meniscus tears were overlooked in the report.

\section{Cruciate ligaments}

Diagnostic accuracy of $97 \%$ (313/324 correct diagnoses) was achieved for anterior and posterior cruciate ligaments. Arthroscopy detected 30 anterior cruciate ligament tears (prevalence 19\%). 27 of these were correctly identified on MRI ( $\triangleright$ Fig. 2) and there were 3 false-positive results. One patient had a posterior cruciate ligament tear (prevalence $<1 \%$ ) that was correctly visualized with MRI.

\section{Loose joint bodies and medial patellar plicae}

Arthroscopy identified loose joint bodies in 21 cases (prevalence $13 \%) .10$ of these were correctly visualized with MRI. Arthroscopy showed a medial patellar plica in 7 patients (prevalence $4 \%$ ), 4 of which were detected with MRI.

\section{Cartilage}

An overview of the SE, SP, overestimates and underestimates of the degree of chondropathy and the diagnostic accuracy of the shortened protocol with respect to cartilage damage in the individual compartments is provided in $\mathbf{T a b} \mathbf{5}$.

In total, the cartilage surface was correctly evaluated in $733 / 972$ compartments (75\%) The degree of chondropathy was overestimated in 183 cases (19\%) and underestimated in 56 cases (6\%). The SE, SP, PPV, and NPV were $72 \%, 80 \%, 86 \%$, and $61 \%$, respectively.

The degree of chondropathy in the retropatellar compartment was correctly diagnosed in $100 / 162$ cases (62\%) and was overestimated in 61 cases (38\%). The degree of cartilage damage was underestimated on MRI in only one patient $(<1 \%)$.

The degree of chondropathy in the trochlear groove was correctly diagnosed in $135 / 162$ cases (83\%), was overestimated in $15 / 162$ cases ( $9 \%$ ), and was underestimated in $12 / 162$ cases (7\%).

The degree of damage to the medial femoral joint surface was correctly classified in $131 / 162$ cases $(81 \%)$. The degree of cartilage damage was overestimated in 25 cases (15\%) and was underestimated in 6 cases (4\%). The degree of damage to the lateral cartilaginous coating was correctly classified in $118 / 162$ cases 
- Table 4 Characteristics of the shortened protocol with regard to damage to the meniscus and cruciate ligament, loose joint bodies, and medial patellar plicae.

\begin{tabular}{|l|c|c|c|c|c|}
\hline & SE [\%] & SP [\%] & PPV [\%] & Accuracy [\%] \\
\hline Medial meniscus & 97 & 88 & 94 & 94 & 94 \\
\hline Lateral meniscus & 77 & 99 & 98 & 89 & 91 \\
\hline Anterior cruciate ligament & 90 & 94 & 77 & 98 & 93 \\
\hline Posterior cruciate ligament & 100 & 100 & 100 & 100 & 100 \\
\hline Loose joint bodies & 48 & 96 & 62 & 93 & 90 \\
\hline Medial patellar plicae & 57 & 88 & 18 & 98 & 87 \\
\hline
\end{tabular}
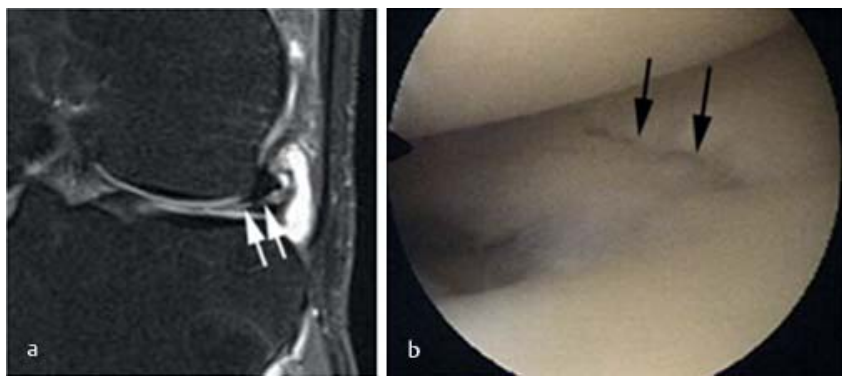

- Fig. 1 Example of a tear running oblique-horizontal to the lower surface in the case of a 58-year-old patient without trauma. a The MRI image (coronal fat-suppressed sequence) shows the tear as a linear increase in signal in the area of the intermediate part (white arrow). b The arthroscopic documentation confirms the finding that the meniscus is significantly damaged with a crossed tear in the intermediate part (black arrows).
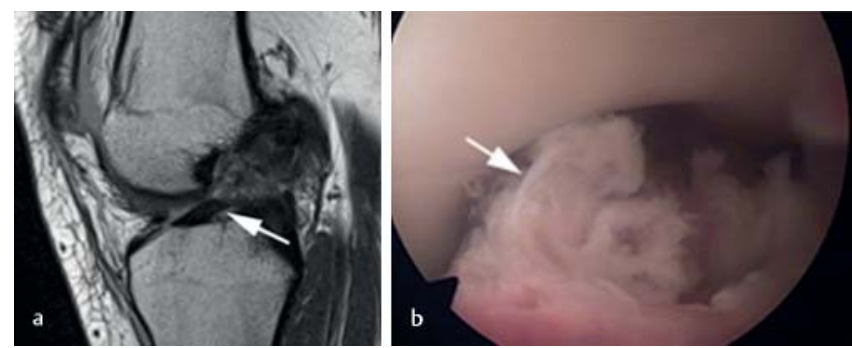

- Fig. 2 Example of a 17-year-old patient with an acute tear of the anterior cruciate ligament caused by indirect trauma while skiing. a The MRI (sagittal proton-density-weighted image) shows no continuity of the cruciate ligament. Only the distal stump is identifiable as a ligamentous structure with low intensity (arrow). b Arthroscopically, the cruciate ligament is inconsistently graspable in the intercondylar notch; only the distal end of the ligament is palpable with a hook probe (arrow).

- Table 5 Characteristics of the shortened protocol with regard to chondral damage.

\begin{tabular}{|l|l|l|l|r|c|}
\hline & SE [\%] & SP [\%] & Accuracy [\%] & Overestimated [\%] & Underestimated [\%] \\
\hline Retro-patellar & 63 & 60 & 62 & 38 & $<1$ \\
\hline Trochlear groove & 76 & 94 & 83 & 9 & 7 \\
\hline Medial femur & 81 & 81 & 81 & 21 & 4 \\
\hline Lateral femur & 70 & 75 & 73 & 17 & 6 \\
\hline Medial tibia & 76 & 80 & 77 & 12 & 6 \\
\hline Lateral tibia & 71 & 92 & 77 & 11 \\
\hline
\end{tabular}

(73\%), was overestimated in 34 cases (21\%), and was underestimated in 10 cases (6\%).

The degree of damage to the medial tibial joint surface was correctly classified in 124/162 cases (77\%) ( $\triangleright$ Fig. 3 ). The degree of cartilage damage was overestimated in 28 cases (17\%) and was underestimated in 6 cases (6\%). The degree of chondropathy in the lateral compartment was correctly classified in 125/162 cases (77\%), was overestimated in 20 cases (12\%), and was underestimated in 17 cases $(11 \%)$ (Figs. $>\mathbf{4}, \mathbf{5})$.

\section{Discussion}

The data shown here prove that knee MRI with a total scan time of $<9$ minutes provides reliable results with high diagnostic value compared to arthroscopy.

The SE and SP of $97 \%$ and $88 \%$, respectively, for the detection of medial meniscus tears and of $77 \%$ and $99 \%$, respectively, for the detection of lateral meniscus tears are comparable with the values specified in the literature. In a recently published metaanalysis regarding the diagnostic accuracy of MRI for the detection of meniscus tears, the SE and SP were $89 \%$ and $88 \%$, respectively, for 

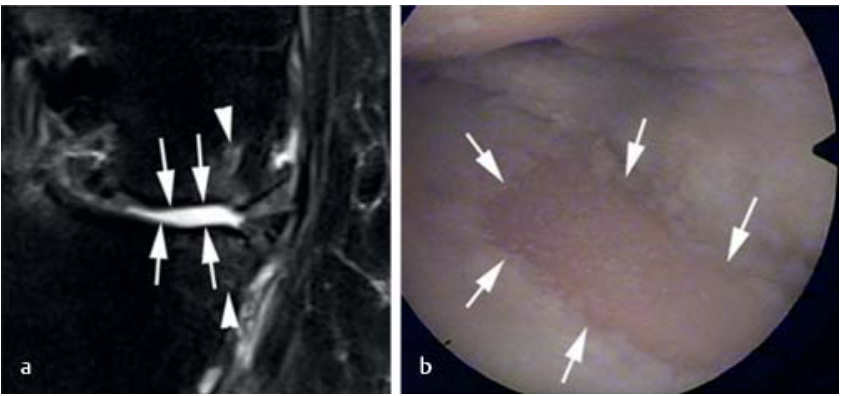

- Fig. 3 Detection of chondral damage in a 46-year-old patient without any known trauma. a MRI (coronal proton-density-weighted fat-suppressed image) shows areas of chondropathy grade 3 with diffuse tibial and femoral chondral denudation (arrows). There are also bone marrow edema-like subchondral areas which are only detectable by MRI (arrowheads). b Arthroscopy confirmed the chondral finding. The documentation shows a large tibial area with denudation to the subchondral bone as defined by a grade 3 chondral lesion (arrows).
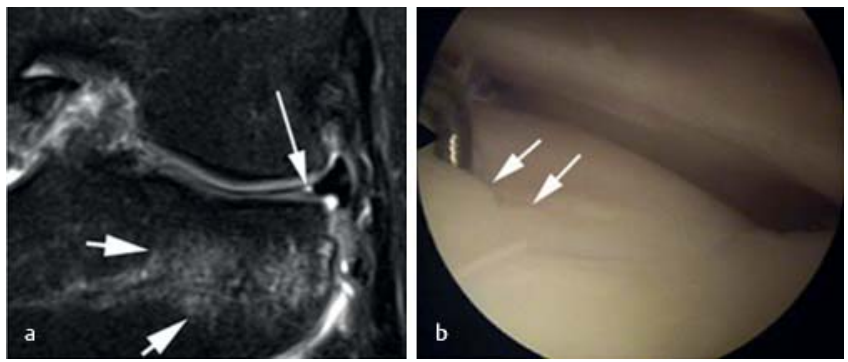

- Fig. 4 Example of a false-negative MRI finding in cartilage evaluation. a MRI (coronal proton-weighted fat-suppressed image). After a trauma incident, a 45 -year-old patient shows an intact surface at the lateral and femoral cartilage. Furthermore, the image shows osseous edema of contusion on the tibial side (short arrows) and a radial meniscus tear (long arrow). b Arthroscopy shows chondropathy grade $2 \mathrm{~B}$. The hook probe sinks in and shows partial chondral delamination (arrow).

the medial meniscus and $78 \%$ and $95 \%$, respectively, for the lateral meniscus [19]. In our study, the majority of undetected tears $(13 / 16=81 \%)$, which were primarily small tears or the total extent of a more complex tear, were still not able to be detected upon reexamination of the MRI images despite knowledge of the arthroscopy findings. This can be explained by the type of primary method, since MRI visualizes the knee as a snapshot in a stationary state and arthroscopy allows functional exploration of an injury via hook probe examination. Linear meniscus tears or branches of the tear that are very fine and lie on top of one another are not detected by MRI, but hook probe examination can identify such tears.

Anterior cruciate ligament tears could be reliably diagnosed with an accuracy of $93 \%$, an SE of $90 \%$, and SP of $94 \%$ in our study. These results are comparable with those in the metaanalysis cited in section regarding menisci. An SE of $87 \%$ and an SP of $93 \%$ for anterior cruciate ligament tears is specified there [19].

The diagnosis of "radiologically symptomatic" medial patellar plicae was only possible on a limited basis with the shortened pro-
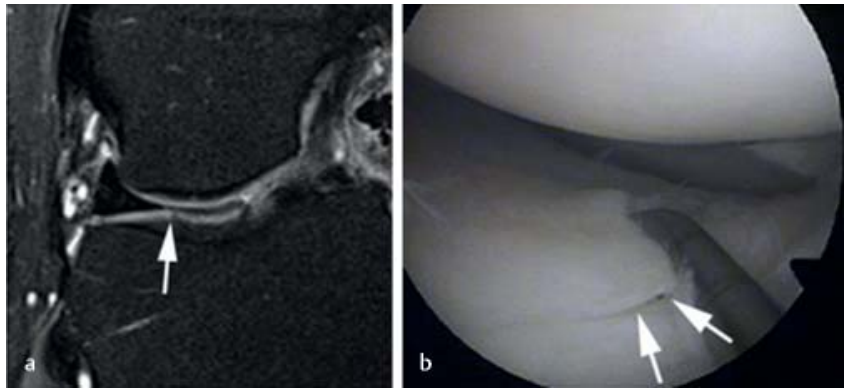

- Fig. 5 55-year-old patient with lateral atraumatic pain. a MRI (coronal proton-weighted fat-suppressed image) shows an intact lateral tibial cartilage surface and only circumscribed intrachondral signal hypointensity as defined by a nonspecific finding (arrow). b Arthroscopy shows chondropathy grade 2B. The hook probe undermines the chondral tear (arrows). With knowledge of the arthroscopy findings, the hypointensity on the MRI image must be retrospectively evaluated as a chondral lesion.

tocol introduced here. Isolated "radiologically symptomatic" medial patellar plicae were not present in our patient population but could be diagnosed as a co-pathology in the majority of cases (57\%). The metaanalysis of Stubbings et al. [20] yielded an SE of $77 \%$ and an SP of $58 \%$ compared to the SE of $57 \%$ and SP of $88 \%$ achieved in our study. The reason for the limited ability to evaluate a medial patellar plica on MRI may be the non-standardized criteria used to diagnose medial patellar plicae. The arthroscopic classification system based on Sakakibara [21] that is generally accepted by radiologists and surgeons performing arthroscopy is primarily used for classification according to the size and position of a medial patellar plica. Characterization of a plica on the basis of its thickness does not correlate significantly with clinical symptoms [22]. The classification of "radiologically symptomatic" plica with concomitant cartilage damage used in this study also did not provide reliable results.

In addition to the evaluation of "radiologically symptomatic" plicae, the evaluation of cartilage damage on MRI continues to be a challenge independent of field strength $[23,24]$ as reflected by our results (SE $72 \%$ and SP $80 \%$ ). Our values are slightly higher than those published by Kijowski et al. [25], who examined 100 patients on a $1.5 \mathrm{~T} \mathrm{MRI} \mathrm{scanner} \mathrm{with} \mathrm{a} 5$-sequence standard protocol (sagittal: Intermediate-weighted T2 FSE; coronal: Intermediate-weighted T1 FSE; transverse: T2 FSE) and a total scan time of $14: 40$ minutes. The SE for the detection of cartilage damage was $69 \%$ and the SP was $78 \%$. The SE of our shortened protocol was comparable to a metaanalysis by Zhang et al. [24] (72\% vs. $75 \%$ ) but the SP was significantly lower ( $80 \%$ vs. $94 \%$ ). The inclusion criteria of the metaanalysis are probably responsible for the comparatively lower values of the shortened protocol. Among other things, studies performed at $3 \mathrm{~T}$ and containing $3 \mathrm{D}$ sequences were included so that these results can only be compared to our study results on a limited basis.

The literature contains only three publications [26-28] with studies comparable to our study using parallel imaging and arthroscopy as the gold standard, two of which were performed in the same patient population. 
Magee et al. [26] examined 34 patients using SMASH (simultaneous acquisition of spatial harmonics) and only T2-weighted sequences. These sequences were compared with a conventional 5-sequence protocol (sagittal: T2 TSE FS; coronal: T1 TSE, T2 TSE FS; axial: T2 TSE FS) and arthroscopy as the gold standard. The total scan time when using SMASH was 6 minutes 38 seconds. The SE and SP for meniscus tears, anterior cruciate ligament tear, and cartilage defects were $100 \%$. Detailed evaluation of the articular cartilage was not performed. In addition to the low number of patients, this may be another reason for the high SE and SP.

In addition to Magee et al., Van Dyck et al. [27, 28] examined patients with parallel acquisition techniques and arthroscopy as the gold standard. This group examined 100 patients and compared their results with arthroscopy as the reference standard. As in our study, an acceleration factor of 2 was used in parallel imaging. The MRI protocol included 4 sequences (sagittal: T2 PD; coronal: T1, T2 PD FS; axial: T2 PD FS) with a total scan time of exactly 9 minutes.

The results were presented in 2 publications with one study addressing meniscus and anterior cruciate ligament injuries [27], and the other addressing cartilage damage [28]. The SE and SP were $93 \%$ and $90 \%$, respectively, for the medial meniscus and $77 \%$ and $99 \%$, respectively, for the lateral meniscus. Our results were comparable for the medial meniscus (SE 97\%, SP $88 \%$ ) and identical for the lateral meniscus (SE 77\%, SP 99\%). Van Dyck et al. specified an SE of $78 \%$ and an SP of $100 \%$ for anterior cruciate ligament injuries. Our study yielded a higher SE of $90 \%$ with a slightly lower SP of $94 \%$. Van Dyck et al. specified an SE of $60 \%$ and an SP of $96 \%$ for the evaluation of articular cartilage. As in the case of the evaluation of the anterior cruciate ligament, our SE results are higher than these values (73\%) with a comparatively lower SP (80\%). The diagnostic accuracy for articular cartilage was $87 \%$ in the study by van Dyck et al. and $75 \%$ in our study. We explain this difference primarily with the relatively low SP in the evaluation of patellar cartilage changes. The degree of damage to an arthroscopically normal cartilaginous coating was often overestimated. The retrospective analysis showed that signal changes were incorrectly evaluated as surface defects.

\section{Limitations of the study}

The surgeon performing arthroscopy was not blinded to the results of the previously performed MRI examination. In the case of a clinical discrepancy between a normal MRI finding and a pathological clinical finding, the surgeon was free to perform arthroscopy. This was the case in only $3 / 162$ patients ( $2 \%$ so that it does not affect the conclusions of this study.

A further limitation is that a direct sequence protocol comparison was not possible since scan times were only available on a limited basis.

"Diagnostic" arthroscopy to verify MRI findings was not performed for all 706 consecutive patients. This was not possible for ethical reasons. Moreover, the MRI data were evaluated by only one radiologist. However, since MRI findings are generated by "only" one radiologist in the clinical routine, our results can be effectively compared to the clinical routine.
Our patient population included both traumatic and degenerative injuries. A further differentiation in this regard was not performed in the present study which represents a limitation.

The protocol used in this study can only be applied on a limited basis for the dedicated evaluation of bone marrow infiltration by infectious or tumorous processes, since no T1 SE sequence was used. However, this would not result in any relevant differences regarding acquisition time.

A further main limitation is the classification based on Noyes [17] used by radiologists and surgeons performing arthroscopy to evaluate chondral damage. While radiologists use only the chondral thickness and signal homogeneity to assess chondral damage, surgeons performing arthroscopy can additionally evaluate the consistency of the cartilage via hook probe examination. However, this is only relevant for grade 1 lesions. In addition, the surface expansion of the cartilage damage was not recorded radiologically or arthroscopically.

Moreover, potentially clinically relevant changes to the subchondral bone were not evaluated on MRI, representing a limitation. These changes, especially osteochondral and subchondral fractures that are difficult or impossible to detect with arthroscopy, affect prognosis, particularly in a post-traumatic situation $[29,30]$.

The final limitation is the relatively long time period between MRI and arthroscopy, which, in principle, means that it is possible for additional pathologies that cannot be detected on MRI to have occurred in the meantime.

\section{Conclusion}

With the help of parallel imaging, the knee MRI protocol at $1.5 \mathrm{~T}$ presented in our study with a total scan time of $<9$ minutes has high diagnostic value compared to arthroscopy and provides reliable results in the clinical routine.

\section{CLINICAL RELEVANCE OF THE STUDY}

A shortened protocol for knee joint MRI at $1.5 \mathrm{~T}$ with a total scan time $<9$ minutes and parallel imaging has high diagnostic value compared to arthroscopy.

Compared to other studies, the shortened protocol yields comparable results for the menisci and cruciate ligaments. There is a tendency to overestimate the degree of damage when detecting chondral damage.

\section{Disclosure}

The present study was conducted in accordance with the requirements for receiving the academic title of "Dr. med.". 


\section{Conflicts of Interest}

Matthias Stefan May, Rolf Janka, Wolfgang Wuest and Michael Uder are part of the speakers' bureau of Siemens AG, Michael Uder is part of the speakers' bureau of Bracco Imaging $\mathrm{GmbH}$.

\section{References}

[1] Runkel M, Kreitner KF, Regentrop H] et al. Treffsicherheit der Magnetresonanztomografie zum Nachweis von Meniskusrissen. Unfallchirurg 2000; 103: 1079-1085

[2] Behairy NH, Dorgham MA, Khaled SA. Accuracy of routine magnetic resonance imaging in meniscal and ligamentous injuries of the knee: comparison with arthroscopy. Int Orthop 2009; 33: 961-967

[3] De Smet AA, Blankenbaker DG, Kijowski R et al. MR diagnosis of posterior root tears of the lateral meniscus using arthroscopy as the reference standard. Am J Roentgenol 2009; 192: 480-486

[4] Teng CD, Qiu QD. The value of MRI in diagnosis of ligament injuries of knee joint. Zhongguo Gu Shang 2010; 23: 755 -758

[5] Valles-Figueroa JF, Malacara-Becerra M, Villegas-Paredes P et al. Comparison of MRI and arthroscopic images for the diagnosis of knee disorders. Acta Ortop Mex 2010; 24: 8-13

[6] Rappeport ED, Mehta S, Wieslander SB et al. MR imaging before arthroscopy in knee joint disorders? ActaRadiol 1996; 37: 602-609

[7] Crawford R, Walley G, Bridgman S et al. Magnetic resonance imaging versus arthroscopy in the diagnosis of knee pathology, concentrating on meniscal lesions and ACL tears: a systematic review. Br Med Bull 2007; 84: $5-23$

[8] Harris JD, Brophy RH, Jia G et al. Sensitivity of magnetic resonance imaging for detection of patellofemoral articular cartilage defects. Arthroscopy 2012; 28: $1728-1737$

[9] Monroe JW, Schmalbrock P, Spigos DG. Phased array coils for upper extremity. Magn Reson Med 1995; 33: 224-229

[10] Alley MT, Grist TM, Swan JS. Development of a phased-array coil for the lower extremities. Magn Reson Med 1995; 34: 206-267

[11] Kwok WE, Lo KK, Seo G et al. A volume adjustable four-coil phased array for high resolution MR imaging of the hip. MAGMA 1999; 9: 59-64

[12] Kreitner KF, Romaneehsen B, Krummenauer $F$ et al. Fast magnetic resonance imaging of the knee using a parallel acquisition technique (mSENSE): a prospective performance evaluation. Eur Radiol 2006; 16 : $1659-1666$

[13] Doria AS, Chaudry GA, Nasui C et al. The use of parallel imaging for MRI assessment of knees in children and adolescents. PediatrRadiol 2010; 40: $284-293$

[14] Zuo J, Li X, Banerjee $S$ et al. Parallel imaging of knee cartilage at 3 Tesla. J Magn Reson Imaging 2007; 267: 1001 - 1009
[15] Nguyen JC, De Smet AA, Graf BK et al. MR Imaging-based Diagnosis and Classification of Meniscal Tears. RadioGraphics 2014; 34: 981 - 999

[16] De Smet AA, Tuite MJ. Use of the "Two-Slice-Touch" Rule for the MRI Diagnosis of Meniscal Tears. Am J Roentgenol 2006: 187:912

[17] Noyes FR, Stabler CL. A system for grading articular cartilage lesions at arthroscopy. Am J Sports Med 1989; 17: 505-513

[18] Robertson PL, Schweitzer ME, Bartolozzi AR et al. Anterior cruciate ligament tears: evaluation of multiple signs with MR imaging. Radiology 1994; 193: 829-834

[19] Phelan N, Rowland P, Galvin R et al. A systematic review and Meta-analysis of the diagnostic accuracy of MRI for suspected ACL and meniscal tears of the knee. KneeSurg Sports TraumatolArthros 2016; 24: 1525 1539

[20] Stubbings N, Smith T. Diagnostic test accuracy of clinical and radiological assessments for medial patella plica syndrome: A systematic review and meta-analysis. The Knee 2014; 21: 486-490

[21] Sakakibara J. Arthroscopic study on lino's band (plica synovialis mediopatellaris). JJpnOrthop Ass 1976; 50: 513-522

[22] Weckström M, Niva MH, Lamminen A et al. Arthroscopic resection of medial plica of the knee in young adults. Knee 2010; 17: 103-107

[23] Kohl S, Meier S, Ahmad S et al. Accuracy of cartilage-specific 3-Tesla 3D-DESS magnetic resonance imaging in the diagnosis of chondral lesions: comparison with knee arthroscopy. J OrthopSurg Res 2015; 10: 191

[24] Zhang M, Min Z, Rana N et al. Accuracy of magnetic resonance imaging in grading knee chondral defects. Arthroscopy 2013; 29: 349-356

[25] Kijowski R, Blankenbaker DG, Davis KW et al. Comparison of 1.5- and 3.0-T MR imaging for evaluating the articular cartilage of the knee joint. Radiology 2009; 250: 839-848

[26] Magee T, Shapiro M, Williams D. Usefulness of Simultaneous Acquisition of Spatial Harmonics Technique for MRI of the Knee. Am J Roentgenol 2004; 182: $1411-1415$

[27] Van Dyck P, Vanhoenacker FM, Lambrecht V et al. Comparison of 1.5 and 3.0-T MRI for Evaluating the Knee Menisci and ACL. J Bone Joint Surg Am 2013; 95: 916-924

[28] Van Dyck P, Kenis C, Vanhoenacker F et al. Comparison of 1.5- and 3-T MR imaging for evaluating the articular cartilage of the knee. Knee Surg Sports TraumatolArthrosc 2014; 22: 1376-1384

[29] Kijowski R, Sanogo ML, Lee KS et al. Short-term clinical importance of osseous injuries diagnosed at MR imaging in patients with anterior cruciate ligament tear. Radiolgy 2012; 264: 531 - 541

[30] Van Meer BL, Oei EH, Meuffels DE et al. Degenerative Changes in the Knee 2 Years After Anterior Cruciate Ligament Rupture and Related Risk Factors: A Prospective Observational Follow-up Study. AM J Sports Med 2016; 44: $1524-1533$ 